\title{
Large-scale velocity structures in turbulent thermal convection
}

\author{
X.-L. Qiu and P. Tong* \\ Department of Physics, Oklahoma State University, Stillwater, Oklahoma 74078
}

(Received 16 February 2001; published 29 August 2001)

\begin{abstract}
A systematic study of large-scale velocity structures in turbulent thermal convection is carried out in three different aspect-ratio cells filled with water. Laser Doppler velocimetry is used to measure the velocity profiles and statistics over varying Rayleigh numbers $\mathrm{Ra}$ and at various spatial positions across the whole convection cell. Large velocity fluctuations are found both in the central region and near the cell boundary. Despite the large velocity fluctuations, the flow field still maintains a large-scale quasi-two-dimensional structure, which rotates in a coherent manner. This coherent single-roll structure scales with $\mathrm{Ra}$ and can be divided into three regions in the rotation plane: (1) a thin viscous boundary layer, (2) a fully mixed central core region with a constant mean velocity gradient, and (3) an intermediate plume-dominated buffer region. The experiment reveals a unique driving mechanism for the large-scale coherent rotation in turbulent convection.
\end{abstract}

DOI: 10.1103/PhysRevE.64.036304

PACS number(s): 47.27.Te, 82.70.Dd, 05.40.-a, 83.10.Pp

\section{INTRODUCTION}

In the study of turbulent thermal convection, much attention is focused on the scaling behavior of temperature and velocity fluctuations over varying Rayleigh numbers $[1,2]$. The discovery of scaling laws in the heat flux and temperature statistics $[3,4]$ has stimulated considerable theoretical efforts [2,4,5], aimed at explaining the observed scaling laws. The theoretical calculations arrive at similar conclusions for the temperature field but have different assumptions and predictions for the velocity field in the bulk region and near the cell boundaries. Direct measurements of the velocity field, therefore, become important to verify assumptions and test predictions of the theoretical models $[2,4,5]$. In contrast to the great number of temperature measurements, however, experimental information about the structure and dynamics of the velocity field in turbulent convection is rather limited.

Experimental studies of the velocity field are difficult because of the severe fluid conditions encountered in convection. Over the past decades, many experimental methods were used to measure the velocity field in turbulent convection and have achieved various degrees of success. Early measurements [6-9] provided useful order-of-magnitude estimates for the velocity fluctuations, but the results were largely semiquantitative. In recent years, several new methods were developed to overcome the experimental difficulties $[10-16]$ and have been used to measure the velocity fluctuations in the central region and the time-averaged velocity profile near the cell boundaries. While these studies are useful in determining the scaling properties of the velocity fluctuations at a few specific locations in the convection cell, the overall flow structure of turbulent convection has remained elusive.

Because of the large number of convecting fluids used in the experiment and the extensive variations of fluid properties and experimental control parameters, one has not been able to find a velocimetry method that can be used widely in convection experiments. The velocimetry methods used so

\footnotetext{
*Email address: ptong@okstate.edu
}

far can measure certain aspects of the flow field but have various degrees of limitations either in the accuracy of the velocity measurement or in the applicability to the convecting fluids. Understanding the applicability and the limitation of these techniques is very important for correct data analysis and interpretation of the experimental results. We now briefly review several velocimetry methods, which have been used in the study of thermal turbulence. The main purpose of the review is to compare the differences among the velocimetry techniques and discuss their limitations when used in turbulent thermal convection. One needs to be aware of these limitations when designing an experiment and they may or may not apply to the particular experiments mentioned in the following discussion.

The early experiments by Deardorff and Willis [7] and later by Fitzjarrald [8] used a moving hot-wire anemometer to measure the flow velocity in turbulent convection of air. The flow velocity was obtained from the electric signals needed to keep two crossed hot wires at a constant temperature. Strong temperature fluctuations in the convecting fluid introduce additional noise to the measured electric signals and thus severely affect the accuracy of the hot-wire anemometer. The large temperature fluctuations may ruin the calibration of the hot-wire anemometer, making the technique unsuitable for thermal turbulence.

The experiment by Garon and Goldstein [6] used the laser Doppler velocimetry (LDV) technique to measure the rms velocity profile along the central axis of the convection cell and the velocity fluctuations at the cell center over varying Rayleigh numbers up to $\mathrm{Ra} \simeq 2.5 \times 10^{9}$. Because the Bragg cell was not available at the time to shift the frequency of one of the incident laser beams, only the absolute value of the vertical velocity fluctuations was obtained. As a result, the measured rms velocity profile was contaminated by the varying mean velocity in the flow. In addition, the measured rms velocities were biased towards the large values, because of the instrumentation cutoff for small velocities near zero. It was noticed in the experiment that a long-time average is needed in order to obtain a steady value for the rms velocity. It will be shown below that the velocity field in turbulent convection has relatively large fluctuations and small mean 
values, which drift slowly with time. Long-time continuous measurements are, therefore, required at each spatial location in order to obtain adequate statistics for the velocity measurements.

Tanaka and Miyata [9] used a photographic technique to measure the velocity fluctuations in the central region of the cell over varying Rayleigh numbers up to $\mathrm{Ra} \simeq 4 \times 10^{9}$. In the experiment, hydrogen bubbles were used as tracer particles and only a few long trajectories of the hydrogen bubbles were sampled to calculate the flow velocity. No long-time average was performed in the measurements. Because the flow velocity is small, the rising velocity of the hydrogen bubbles due to their own buoyancy becomes significant and affects the accuracy of the velocity measurement considerably. To obtain the rms velocity, Tanaka and Miyata also assumed that the velocity fluctuations in the central region are homogeneous and isotropic, which is not valid for largeaspect-ratio convection cells, as will be shown below.

In a low-temperature helium convection experiment, Sano, $\mathrm{Wu}$, and Libchaber [10] measured the large-scale flow velocity near the sidewall of the cell over varying Ra up to $\mathrm{Ra} \sim 10^{12}$. By measuring the cross-correlation function between two temperature signals, they obtained the average transit time of the temperature fluctuations (thermal plumes) across two adjacent temperature probes. The flow velocity is determined by the distance between the two temperature probes divided by the transit time. This method was also used later in several other convection experiments using either low-temperature helium gas $[17,18]$ or mercury $[19]$ as the working fluid. Like many other time-of-flight velocimeters, however, the dual-probe method is sensitive only to large velocity fluctuations.

Because of turbulent velocity fluctuations, the large temperature fluctuations (or thermal plumes) travel not only in the direction parallel to the connecting line between the two temperature probes but also in the transverse directions. When the transit time across the two probes becomes longer than that across the lateral dimension of a single probe (or of a thermal plume), the temperature fluctuation will not cross the two probes in succession and hence their motion cannot be detected by the cross-correlation scheme. By equating the two transit times, one finds the cutoff velocity $v_{c}$ $\simeq \sigma\left(\ell / r_{0}\right)$, where $\sigma$ is the rms velocity, $\ell$ is the spatial separation between the two temperature probes, and $r_{0}$ is the larger lateral dimension of either the temperature probe or the thermal plume. In the experiment by Sano et al. [10], $\ell \simeq 2 \mathrm{~mm}$ and $r_{0} \simeq 0.2 \mathrm{~mm}$ (probe size), and hence we have $v_{c} \simeq 10 \sigma$. If we use the plume size $\sim 1 \mathrm{~mm}$ (the thermal boundary layer thickness at $\mathrm{Ra} \simeq 10^{9}$ ) as the typical value of $r_{0}$, we find $v_{c} \simeq 2 \sigma$. Clearly, the dual-probe method works only in the region where the mean velocity is much larger than its standard deviation.

Instead of using two temperature probes, Xin, Xia, and Tong $[13,20]$ developed a dynamic light scattering method to measure the transit time of small seed particles in water to cross two parallel laser beams. With this technique, they measured the velocity boundary layer profile near a conducting surface in the $\mathrm{Ra}$ range $10^{8}<\mathrm{Ra}<10^{10}$. Because the laser beams are noninvasive, their separation $\ell$ can be set very small $(\ell \simeq 0.2 \mathrm{~mm}$ ). As a result, the cutoff velocity is reduced to $v_{c} \simeq 2.2 \sigma$. As will be shown below, in the boundary layer region the longitudinal velocity component $u$ is much larger than the normal velocity component $v$, and therefore the effect of $v_{c}$ on the measured $u$ is small.

In contrast to the situation near the boundary, the effect of the cutoff velocity $v_{c}$ becomes significant in the central region of the cell, where the velocity fluctuations become larger than their mean values. To solve this problem, Shen, Xia, and Tong [12] used the technique of single-beam twocolor cross-correlation spectroscopy [21] to measure the velocity fluctuations at the cell center, where the mean velocity is zero. The single-beam method uses the intensity crosscorrelation function to resolve the beam crossing time. It should be mentioned that both the dual-beam (or dualtemperature-probe) method and the single-beam method use the time-averaged cross-correlation function $g(\tau)$ to resolve the time of flight. Consequently, information about the velocity statistics is convoluted in the measured $g(\tau)$. The obtained velocity parameters from the curve fitting, therefore, become model dependent.

In a study of the temperature and velocity profiles of turbulent convection in water, Tilgner, Belmonte, and Libchaber [11] used an imaging method to determine the traveling distance of electrochemically labeled particles of fluid in a given time. Because the imaging method requires manual accumulation, the velocity measurements were conducted only at a single value of $\mathrm{Ra}\left(\simeq 10^{9}\right)$. While it is adequate to obtain a good velocity profile, the imaging technique is rather limited by its low sampling rate and poor velocity statistics.

Recently, Ashkenazi and Steinberg [16] studied turbulent convection in compressed $\mathrm{SF}_{6}$ near its gas-liquid critical point. Critical density fluctuations of the fluid scatter light strongly and were used as the "seed particles" for the LDV measurement. Unlike the solid seed particles, however, the internal dynamics of the critical density fluctuations in turbulent convection may introduce additional noise to the LDV measurement. In fact, the frequency shift $\Omega$ measured in this case has three contributions: $\Omega=\mathbf{q} \cdot \mathbf{v}+\omega_{e q}+\omega_{t u r}$, where $\mathbf{q} \cdot \mathbf{v}$ is the Doppler beat frequency associated with the translational motion of the fluid droplet with $\mathbf{v}$ being the local velocity and $\mathbf{q}$ the photon momentum transfer vector. The frequency shift $\omega_{e q}$ is due to the (equilibrium) critical fluctuations at a fixed temperature $T$, and $\omega_{t u r}$ is an additional contribution due to the temperature fluctuations in turbulent convection.

Ashkenazi and Steinberg argued that $\omega_{e q}$ is very small when compared with $\mathbf{q} \cdot \mathbf{v}$, but they ignored the important contribution of $\omega_{t u r}$. Because of the strong dependence of the fluid properties on the effective temperature $T-T_{c}$, where $T_{c}$ is the critical temperature of the gas-liquid transition, $\omega_{t u r}$ is strongly correlated with $\mathbf{q} \cdot \mathbf{v}$ in time and may become very large when $T-T_{c}$ is small. As a result, the $\mathrm{LDV}$ measurements in $\mathrm{SF}_{6}$ could be contaminated by the strong temperature fluctuations in turbulent convection. While singular behavior of the fluid properties near the critical point offers opportunities to vary the Rayleigh number 
and the Prandtl number in a wide range $\left(10^{9}<\mathrm{Ra}<10^{14}, 1\right.$ $<\operatorname{Pr}<100$ ), great care must be exercised because all the fluid properties become ill-defined when the temperature fluctuations in turbulent convection are comparable to $T$ $-T_{c}$.

In this paper, we present a comprehensive study of largescale velocity structures in turbulent thermal convection. The experiment is conducted in three different aspect-ratio cells filled with water. The technique of LDV is used to measure the mean velocity profiles and the velocity statistics over varying $\mathrm{Ra}$ and spatial positions across the whole convection cell. The fiber-optic LDV is a matured technique with high accuracy (better than 1\%) and fast sampling rate. It is nonintrusive and is capable of measuring the local velocity over a long period of time. In the experiment to be described below, we measure the local velocity over a long period of time up to $7-8 \mathrm{~h}$ at each spatial location. More than 2600 laser hours are spent in total to obtain a complete series of velocity data at various Rayleigh numbers and spatial locations with the highest statistical accuracy possible. These velocity measurements allow us to fully characterize the largescale flow structure in turbulent convection and answer some important open questions that are related directly to the physical understanding of convective turbulence. These questions include: How is the large-scale circulation, that spans the height of the cell, generated and sustained? What is the connection between the large-scale circulation and the thermal plumes that erupt from the upper and lower boundary layers?

While LDV is an ideal technique for the investigation attempted here, it can be used only in water and some other common solvents, in which one can readily seed a small amount of micron-sized particles. These particles follow the local flow and at the same time scatter the incident laser light. In the convection experiments using low-temperature helium gas $[4,17,18]$, room-temperature compressed gases $[22]$, or mercury $[19,23]$ as the working fluid, particle seeding is extremely difficult, preventing one from using LDV and other light scattering methods to measure the flow velocity in these systems. Unlike turbulent convection in a gas, in which one can vary $\mathrm{Ra}$ for many decades, the variable range of $\mathrm{Ra}$ in a liquid system is rather limited (typically $1-2$ decades), because the liquid density cannot be changed very much. Large fluctuations of the fluid refractive index resulting from temperature fluctuations may cause the two laser beams used in LDV to wander and defocus in the convecting fluid. The corruption of laser beam properties reduces the signal-to-noise level of LDV. However, this beam corruption occurs mainly near the upper and lower thermal boundary layers, whose thickness is much smaller than that of the viscous boundary layers for large Prandtl number fluids, such as water. The thermal boundary layer thickness in water is less than $1 \mathrm{~mm}$ when $\mathrm{Ra}>10^{9}$.

The remainder of the paper is organized as follows. We first describe the apparatus and the experimental method in Sec. II. Experimental results are discussed in Sec. III. Finally, the work is summarized in Sec. IV.

\section{EXPERIMENT}

The experiment is conducted in a vertical cylindrical cell filled with water. Details about the apparatus have been described elsewhere [24] and here we only mention some key points. The side-wall of the cell is made of transparent Plexiglas with an inner diameter of $19 \mathrm{~cm}$ and wall thickness of $0.63 \mathrm{~cm}$. Three Plexiglas rings with the same diameter but having three different heights, $9.5,20.3$, and $40.7 \mathrm{~cm}$, are used respectively. The corresponding aspect ratios (A $=$ diameter/height) of the cell are $A \simeq 2,1$, and 0.5 . The upper and lower plates are made of brass and their surfaces are electroplated with a thin layer of gold. Two silicone rubber film heaters are connected in parallel and sandwiched on the backside of the lower plate to provide a constant and uniform heating. The upper plate is in contact with a cooling chamber, whose temperature is maintained constant by circulating cold water from a temperate bath. The temperature difference $\Delta T$ between the upper and lower plates are measured by two thermistors embedded in each plate.

The experimental control parameter is the Rayleigh number $\mathrm{Ra}=\alpha g \Delta T L^{3} /(\nu \kappa)$, where $\mathrm{g}$ is the gravitational acceleration, $\mathrm{L}$ is the height of the cell, and $\alpha, \nu$, and $\kappa$ are, respectively, the thermal expansion coefficient, the kinematic viscosity, and the thermal diffusivity of the convecting fluid (water). In the experiment, the average temperature of the bulk fluid is maintained at $30^{\circ} \mathrm{C}$ and the corresponding Prandtl number, $\operatorname{Pr}=\nu / \kappa$, is fixed at $\sim 5.4$. The temperature of the upper and lower plates is regulated within $0.1{ }^{\circ} \mathrm{C}$ in standard deviation, which is less than $2.5 \%$ of the maximum $\Delta T$ used in the experiment. To measure the local temperature fluctuations of the fluid, a stainless steel tube of diameter 1.1 $\mathrm{mm}$ is installed through the sidewall to guide a small thermistor into the cell. The tube is mounted on a micrometercontrolled translation stage, such that the local temperature can be measured at various horizontal positions along the cell diameter at the mid-height of the cell. The movable thermistor is calibrated with an accuracy of $0.01^{\circ} \mathrm{C}$. A Keithley multimeter is used to measure the resistance of the thermistor at a sampling rate of $20 \mathrm{~Hz}$.

Local velocity measurements are conducted by using a two-component LDV system (TSI Inc.) together with an argon-ion laser (Coherent Innova 90). A long rectangular flat window, $3 \mathrm{~cm}$ in width and having the same height as the Plexiglas ring, is inserted onto the Plexiglas rings to admit the incident laser beams and observe the light scattered by the seed particles. The LDV system has a fiber-optic transceiver, which collects the scattered light in the backward direction and feeds it to two photomultiplier tubes via an optical fiber. The cylindrical probe volume viewed by the photodectors is $1.31 \mathrm{~mm}$ in length and $0.09 \mathrm{~mm}$ in diameter. The analog signals from the photomultiplier tubes are fed to a LDV signal processor (Model IFA-655, TSI Inc.), which uses the fast Fourier transform (FFT) to resolve two components of the flow velocity. Monodisperse polymer latex spheres of $4.75 \mu \mathrm{m}$ in diameter are used as the seed particles. Because the density of the particles $\left(1.05 \mathrm{~g} / \mathrm{cm}^{3}\right)$ 


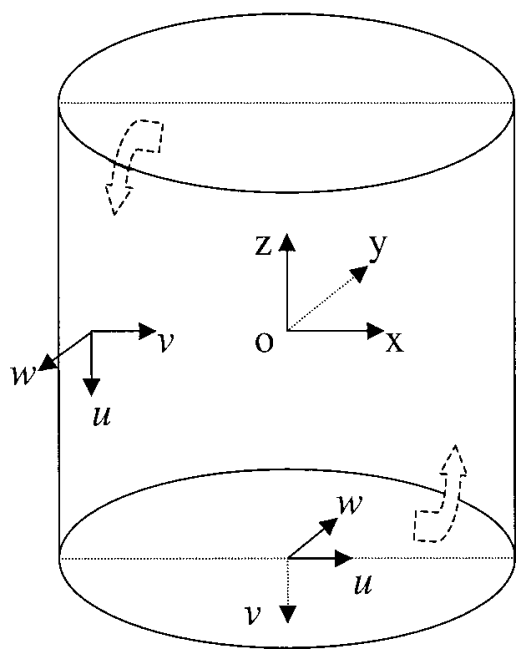

FIG. 1. Space and velocity coordinates used in the presentation of the velocity measurements. The long arrows near the sidewall of the convection cell indicate the direction of the large-scale circulation.

matches closely that of water, they follow the local flow well.

In the experiment, we vary the measuring position of the local velocity by moving the transceiver probe, which is mounted on a traversable table. The sampling rate of the velocity measurements is $10-15 \mathrm{~Hz}$, which is approximately ten times larger than the cutoff frequency of the velocity power spectrum. In the calculation of the velocity statistics, we use the transit time weighting [25] to correct for the velocity sampling bias. Typically, we take 2-h-long time series data ( $\sim 10^{5}$ data points) at each spatial position for the velocity profile measurements and 7-h-long time series data ( $\sim 4 \times 10^{5}$ data points) for the velocity histogram and Radependence measurements. This data accumulation time is much longer than the time scale for the large-scale circulation, which is of the order of $1 \mathrm{~min}$, ensuring that the statistical average of the flow properties is adequate.

Figure 1 shows the space and velocity coordinates to be used in the presentation of the velocity measurements. The origin of the space coordinate system is chosen to coincide with the cell center. The $x$ and $z$ axes are in the rotation plane of the large-scale circulation and the $y$ axis is perpendicular to the rotation plane. The symbols $u, v$, and $w$ are used, respectively, to denote the longitudinal, normal, and transversal velocity components near the cell boundaries. As shown in Fig. 1, $u$ and $v$ represent the velocity components in the rotation plane and $w$ is the velocity component perpendicular to it. The rotational speed of the large-scale circulation is described by the mean value of $u$. Early temperature measurements [23] showed that the azimuth of the large-scale circulation rotates slowly in time when the cylindrical cell is leveled perfectly. To pin down the azimuthal rotation, we tilted the cell by a small angle $\left(<1^{\circ}\right)$. Ciliberto et al. [26] showed that such a small tilt does not affect turbulent convection very much.
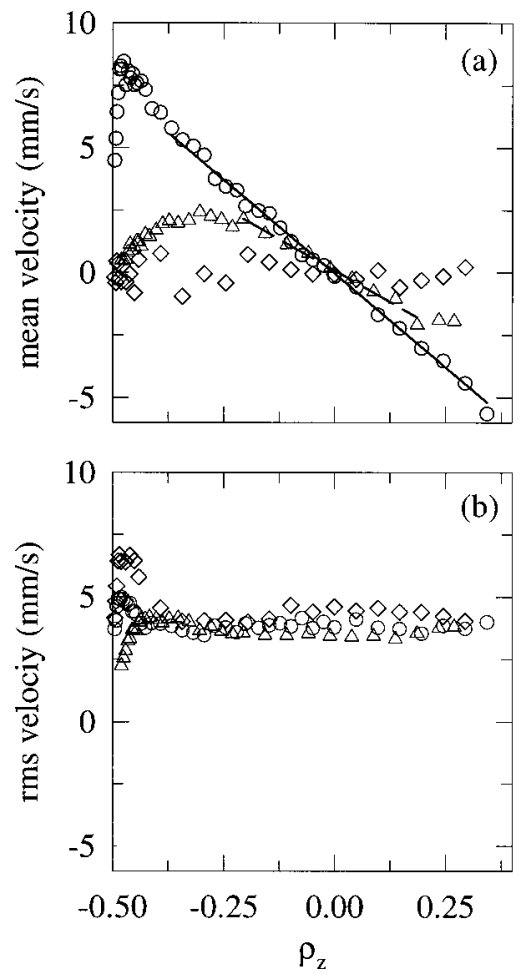

FIG. 2. (a) Time-averaged velocity components, $\bar{u}$ (circles), $\bar{v}$ (triangles), and $\bar{w}$ (diamonds), and (b) their standard deviations, $\sigma_{u}$ (circles), $\sigma_{v}$ (triangles), and $\sigma_{w}$ (diamonds), as a function of the normalized vertical position $\rho_{z}=z / L$. The measurements are made in the $A=1$ cell at $\mathrm{Ra}=3.7 \times 10^{9}$. The solid and dashed lines in (a) are the linear fits to the measured $\bar{u}$ (circles) and $\bar{v}$ (triangles), respectively.

\section{RESULTS AND DISCUSSION}

\section{A. Velocity profiles in various aspect-ratio cells}

\section{1. $A=1$ cell}

We first dicuss the velocity measurements in the $A=1$ cell. Some of the results have been reported previously in a rapid communication [27]. The velocity profile measurements are conducted along two axes; one is along the central $z$ axis from the bottom of the cell to the top ("vertical scan") and the other is along the cell diameter at the mid-height of the cell ("horizontal scan"). These two axes define the rotation plane of the large-scale circulation. For each velocity scan, we measure three velocity components: $u, v$, and $w$.

Figure 2 shows the time-averaged velocity components, $\bar{u}, \bar{v}$ and $\bar{w}$ [Fig. 2(a)], and their standard deviations, $\sigma_{u}$, $\sigma_{v}$, and $\sigma_{w}$ [Fig. 2(b)], as a function of the normalized vertical position $\rho_{z}=z / L$ (vertical scan). Figure 3 shows the time-averaged velocity components, $\bar{u}$ and $\bar{w}$ (open symbols), and their standard deviations, $\sigma_{u}$ and $\sigma_{w}$ (closed symbols), as a function of the normalized horizontal position $\rho_{x}=x / D$ (horizontal scan). Because of the limitations of the LDV optics, we are unable to measure the normal component $v$ in the horizontal scan. All the measurements are conducted at $\mathrm{Ra}=3.7 \times 10^{9}$. It is seen from Figs. 2 and 3 that the flow 


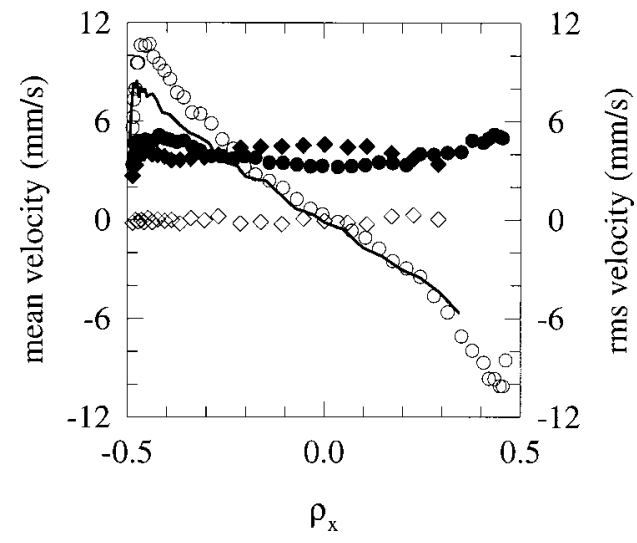

FIG. 3. Time-averaged velocity components, $\bar{u}$ (open circles) and $\bar{w}$ (open diamonds), and their standard deviations, $\sigma_{u}$ (closed circles) and $\sigma_{w}$ (closed diamonds), as a function of the normalized horizontal position $\rho_{x}=x / D$. The measurements are made in the $A=1$ cell at $\mathrm{Ra}=3.7 \times 10^{9}$. The solid curve shows the measured $\bar{u}$ along the $z$ axis for comparison.

field in the $A=1$ cell has a stable, quasi-two-dimensional (quasi-2D) structure. The mean value of the transversal velocity $\bar{w}$ is found to be zero in both the vertical and horizontal scans. The flow field in the rotation plane is divided into three regions: (i) the viscous boundary layer, (ii) the central core region, and (iii) the intermediate buffer region.

In the thin viscous boundary layer $(0.46 \leqslant|\rho| \leqslant 0.5)$, the longitudinal velocity $\bar{u}$ increases linearly with the distance away from the cell boundary and reaches maximum at $|\rho|$ $=\rho_{1} \simeq 0.46$. The normal velocity component $\bar{v}$ increases with $\rho_{z}$ much more slowly and its value at $\left|\rho_{z}\right|=\rho_{1}$ is only $\sim 1 / 8$ of the corresponding $\bar{u}$. Clearly, $\bar{u}$ is the dominant velocity component in the boundary layer and $\bar{v}$ is negligibly small. The rms velocities $\sigma_{u}$ and $\sigma_{w}$ near the conducting surface reach their maximum values of $5 \mathrm{~mm} / \mathrm{s}$ and $6.5 \mathrm{~mm} / \mathrm{s}$, respectively, at $\left|\rho_{z}\right|=\rho_{1}$ and then quickly return to a constant value of $\sim 4 \mathrm{~mm} / \mathrm{s}$ throughout the bulk region [see Fig. 2(b)]. The normal velocity variance $\sigma_{v}$ near the lower surface [see Fig. 2(b)] and the rms velocities $\sigma_{u}$ and $\sigma_{w}$ near the sidewall (see Fig. 3), on the other hand, do not show a sharp peak near the boundary. Rather, they quickly reach a constant value of $\sim 4 \mathrm{~mm} / \mathrm{s}$ and remain unchanged throughout the bulk region. The fact that $\sigma_{u}$ and $\sigma_{w}$ exhibit peak values at $\left|\rho_{z}\right|=\rho_{1}$ indicates that turbulent velocity fluctuations are generated mainly near the upper and lower conducting surfaces, where the thermal plumes are emitted intermittently. It is seen from Figs. 2 and 3 that velocity fluctuations in the viscous boundary layer are small when compared with $\bar{u}$ but are larger than $\bar{v}$. Using a dual-beam light scattering method, Xin et al. [13] have studied the scaling properties of $\bar{u}$ over varying Ra near the viscous boundary layer.

In the central core region $(|\rho|<0.25)$, velocity fluctuations become larger than the mean value. They are approximately homogeneous across the bulk region and nearly isotropic in all three directions. Despite the large velocity fluctuations, the flow field still maintains a coherent structure. The measured $\bar{u}$ is well described by a linear function of the radial distance $\rho$ away from the cell center [solid line in Fig. 2(a)]. The slope of the straight line for $\bar{u}$ remains the same in both the vertical and horizontal scans (see Fig. 3), suggesting that the bulk fluid undergoes a coherent rotation around the cell center.

This large-scale coherent rotation (LSCR) is also observed in flow visualization. In the experiment, we use a photographic technique [24] to take streak pictures of small thermochromic liquid crystal (TLC) spheres seeded in the convecting fluid. A thin vertical sheet of white light is shone through a central section of the convection cell. Figure 4 shows an instantaneous flow field in the $A=1$ cell. A largescale circulation of size comparable to the cell dimension is observed and the 'eye' of the circulation is located approximately at the cell center. It should be noted that the rotation pattern shown in Fig. 4 reveals a special moment of the flow field. A persistent large-scale rotation is found only near the boundary and the flow in the central region looks more irregular and chaotic in other times. This is because the velocity fluctuations in the central region are larger than the mean speed $\bar{u}$. From the flow visualization, we find that the LSCR is not exactly of circular shape. Rather, the rotation is around an ellipse whose long axis is tilted at an angle with respect to the vertical direction (see Fig. 15). Because of this tilt, the measured mean velocity has a small vertical (normal) component $\bar{v}$, which can also be fitted to a linear function of $\rho$ [dashed line in Fig. 2(a)].

The intermediate region between the viscous boundary layer and the central core region is the buffer layer $(0.25$ $<|\rho| \leqslant 0.46$ ), which was also called the mixing zone [4]. In this region, the measured $\bar{v}$ continues to increase with $\rho_{z}$ $=z / L$, whereas the measured $\bar{u}$ starts to decay but still remains larger than $\bar{v}$ [see Fig. 2(a)]. As a result, the normal velocity component $\bar{v}$ becomes increasingly important in the region. The dynamics in the buffer layer is dominated by the mixing and advection of the thermal plumes ejected from the upper and lower thermal boundary layers. Velocity fluctuations in this region remain approximately the same as those in the central region. Their variance is small when compared with $\bar{u}$ but is larger than $\bar{v}$.

\section{2. $A=2$ cell}

Figure 5 shows the time-averaged velocity components, $\bar{u}, \bar{v}$ and $\bar{w}$ [Fig. 5(a)], and their standard deviations, $\sigma_{u}, \sigma_{v}$ and $\sigma_{w}$ [Fig. 5(b)], as a function of $\rho_{z}=z / L$. Figure 6 shows the time-averaged velocity components, $\bar{u}$ and $\bar{w}$ (open symbols), and their standard deviations, $\sigma_{u}$ and $\sigma_{w}$ (closed symbols), as a function of $\rho_{x}=x / D$. All the measurements are conducted at $\mathrm{Ra}=4.9 \times 10^{8}$. It is seen from Figs. 5 and 6 that the flow field in the $A=2$ cell also has a quasi-2D single-roll structure. The transversal velocity component $\bar{w}$ is found to be zero in both the vertical and horizontal scans. The longitudinal velocity component $\bar{u}$ shows a profile similar to that in the $A=1$ cell. The flow field in the rotation plane can still 


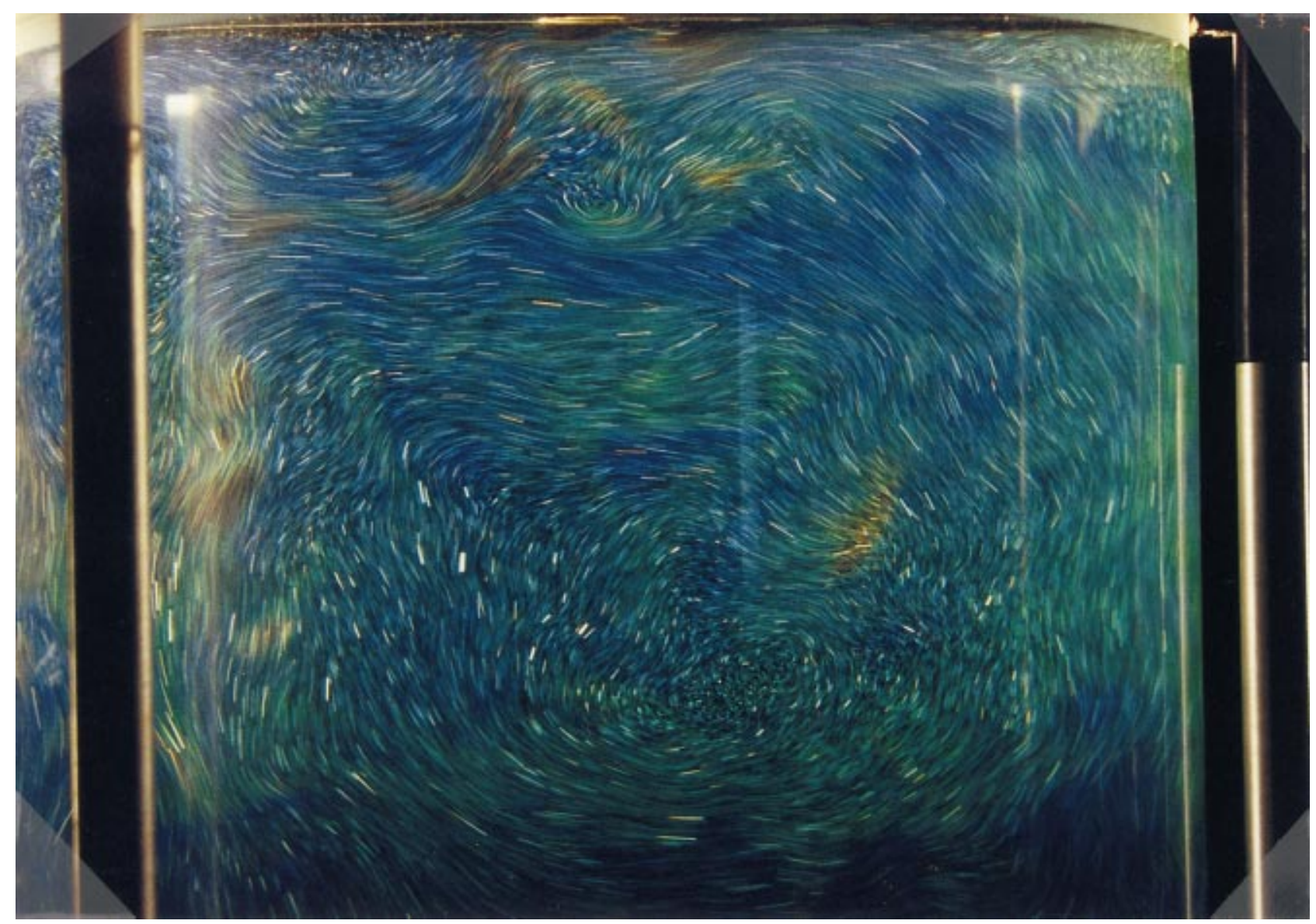

FIG. 4. (Color) A streak image of the TLC spheres taken in the $A=1$ cell at $\mathrm{Ra}=2.6 \times 10^{9}$. Cold eruptions are brown; green and blue regions are warmer. The displayed region covers top $2 / 3$ of the convection cell and its dimension is approximately $20 \mathrm{~cm} \times 14 \mathrm{~cm}$.

be divided into three regions: a thin viscous boundary layer, a central core region with a large-scale coherent rotation (solid line in Fig. 5), and an intermediate buffer region. The measured $\bar{v}$ in the vertical scan is zero, suggesting that the symmetry axes of the LSCR in the $A=2$ cell coincide with the space coordinates shown in Fig. 1.

To further verify the flow structure in the $A=2$ cell, we measure the velocity profile along a vertical axis halfway in between the central $z$ axis and the sidewall ("halfway vertical scan"). The measured $\bar{v}$ is found to increase continously with the distance away from the lower surface and reaches a maximum value at the mid-height of the cell $\left(\rho_{z}=0\right)$. This measurement further confirms the single-roll structure of the LSCR. It is seen from Figs. 5(b) and 6 that the velocity fluctuations in the $A=2$ cell vary with spatial positions and with directions. They are neither isotropic nor homogeneous. This is in contrast with the situation in the $A=1$ cell, in which the velocity fluctuations are approximately homogeneous across the bulk region and nearly isotropic in all three directions.

\section{3. $A=0.5$ cell}

Figure 7 shows the time-averaged velocity components, $\bar{u}, \bar{v}$, and $\bar{w}$ [Fig. 7(a)], and their standard deviations, $\sigma_{u}$, $\sigma_{v}$, and $\sigma_{w}$ [Fig. 7(b)], as a function of $\rho_{z}=z / L$. Figure 8 shows the time-averaged velocity components, $\bar{u}$ and $\bar{w}$ (open symbols), and their standard deviations, $\sigma_{u}$ and $\sigma_{w}$ (closed symbols), as a function of $\rho_{x}=x / D$. All the measurements are conducted at $\mathrm{Ra}=3.28 \times 10^{10}$. It is found that the LSCR in the $A=0.5$ cell is not very stable and wobbles slowly in time when the cell is tilted at the same angle as that for the other two cells. To further stabilize the LSCR, we tilt the $A=0.5$ cell by a larger angle of $1.7^{\circ}$.

The flow field in the $A=0.5$ cell has a similar quasi-2D single-roll structure as that in the $A=1$ cell. In the rotation plane, it still can be divided into three regions: a thin viscous boundary layer, a central core region with a large-scale coherent rotation (solid and dashed lines in Fig. 7), and an intermediate buffer region. Besides these common properties, the velocity field in the $A=0.5$ cell also shows some interesting new features. First, the measured normal component $\bar{v}$ in the central region is larger than the longitudinal component $\bar{u}$, suggesting that the fluid motion is predominantly in the vertical direction. Second, the measured rms velocities are larger than their mean values in the entire region of the cell. As a result, one is unable to visualize a persistent large-scale circulation in the $A=0.5$ cell. As shown in Figs. 7(b) and 8, the measured rms velocities in the 

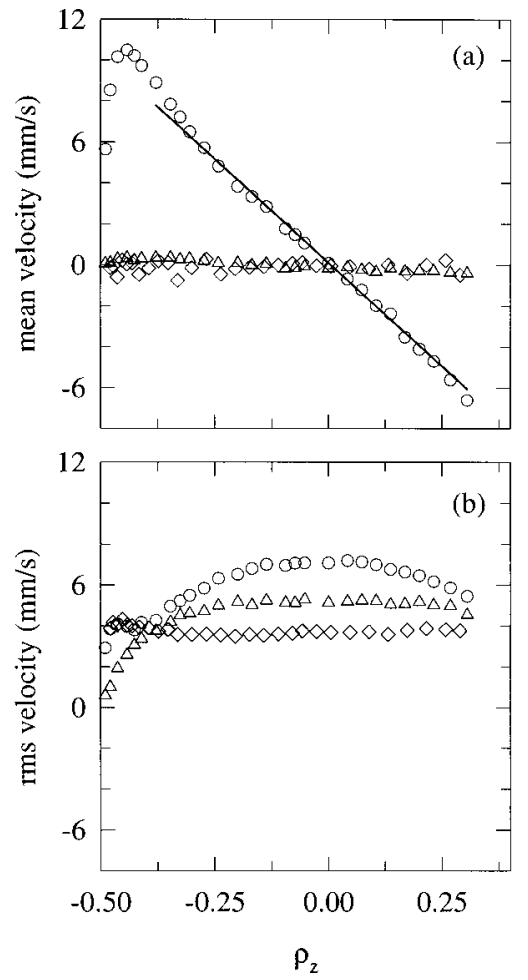

FIG. 5. (a) Time-averaged velocity components, $\bar{u}$ (circles), $\bar{v}$ (triangles), and $\bar{w}$ (diamonds), and (b) their standard deviations, $\sigma_{u}$ (circles), $\sigma_{v}$ (triangles), and $\sigma_{w}$ (diamonds), as a function of the normalized vertical position $\rho_{z}=z / L$. The measurements are made in the $A=2$ cell at $\mathrm{Ra}=4.9 \times 10^{8}$. The solid line in (a) is the linear fit to the measured $\bar{u}$ (circles).

$A=0.5$ cell are more scattered than those in the $A=1$ cell. This is because the LSCR still wobbles somewhat even when the cell is tilted at a larger angle. Previous experiments [28-31] showed that temperature fluctuations in the $A=0.5$

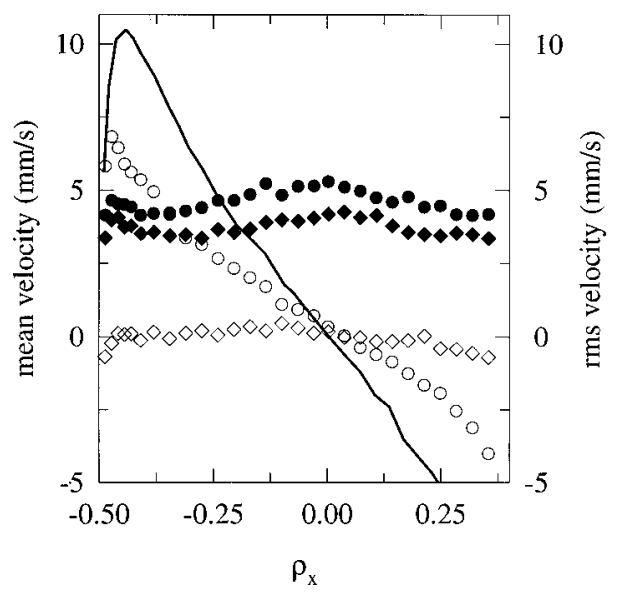

FIG. 6. Time-averaged velocity components, $\bar{u}$ (open circles) and $\bar{w}$ (open diamonds), and their standard deviations, $\sigma_{u}$ (closed circles) and $\sigma_{w}$ (closed diamonds), as a function of the normalized horizontal position $\rho_{x}=x / D$. The measurements are made in the $A=2$ cell at $\mathrm{Ra}=4.9 \times 10^{8}$. The solid curve shows the measured $\bar{u}$ along the $z$ axis for comparison.
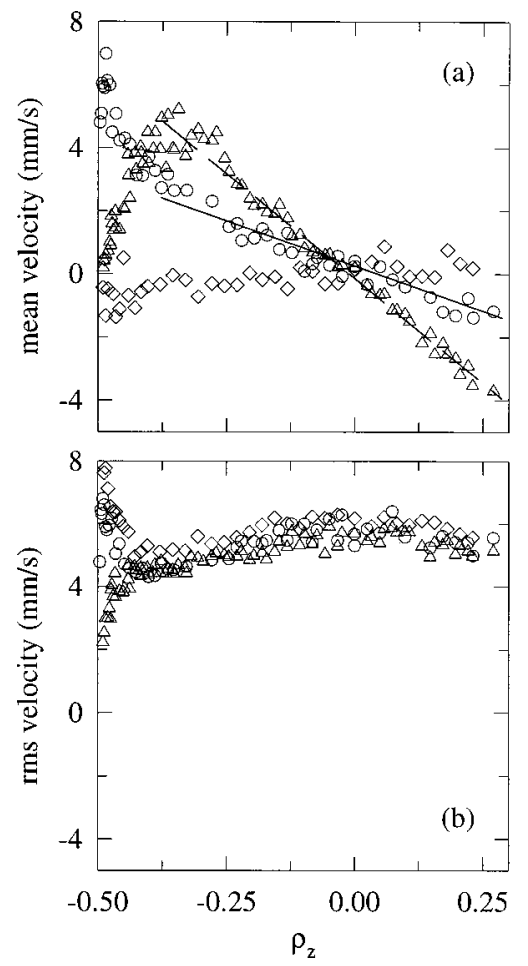

FIG. 7. (a) Time-averaged velocity components, $\bar{u}$ (circles), $\bar{v}$ (triangles), and $\bar{w}$ (diamonds), and (b) their standard deviations, $\sigma_{u}$ (circles), $\sigma_{v}$ (triangles) and $\sigma_{w}$ (diamonds), as a function of the normalized vertical position $\rho_{z}=z / L$. The measurements are made in the $A=0.5$ cell at $\mathrm{Ra}=3.28 \times 10^{10}$. The solid and dashed lines are the linear fits to the measured $\bar{u}$ (circles) and $\bar{v}$ (triangles), respectively.

cell drift slowly with time and their histograms have a nonexponential form. It was suggested $[28,29]$ that these effects are caused by a wobbly LSCR. The velocity measurements shown in Figs. 7 and 8 provide direct evidence to support this argument.

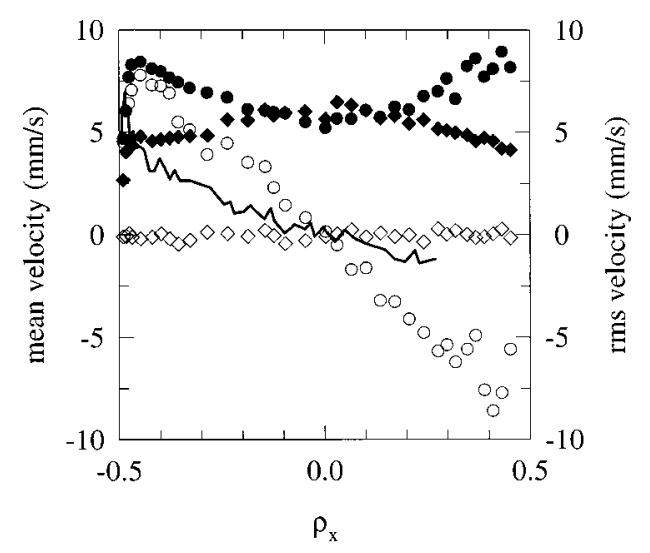

FIG. 8. Time-averaged velocity components, $\bar{u}$ (open circles) and $\bar{w}$ (open diamonds), and their standard deviations, $\sigma_{u}$ (closed circles) and $\sigma_{w}$ (closed diamonds), as a function of the normalized horizontal position $\rho_{x}=x / D$. The measurements are made in the $A=0.5$ cell at $\mathrm{Ra}=3.28 \times 10^{10}$. The solid curve shows the measured $\bar{u}$ along the $z$ axis for comparison. 


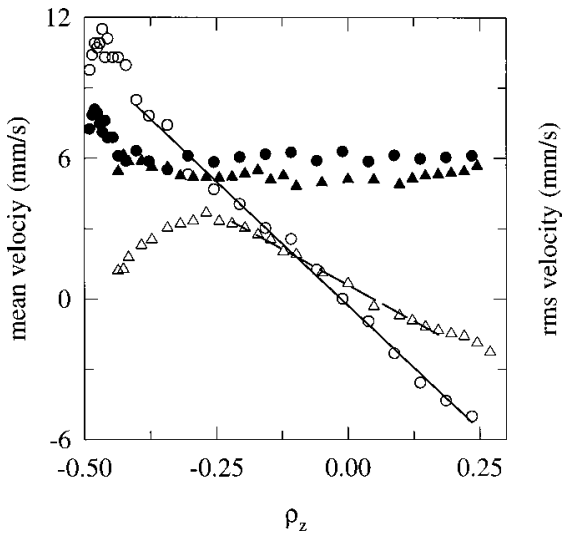

FIG. 9. Time-averaged velocity components, $\bar{u}$ (open circles) and $\bar{v}$ (open triangles), and their standard deviations, $\sigma_{u}$ (closed circles) and $\sigma_{v}$ (closed triangles), as a function of the normalized vertical position $\rho_{z}=z / L$. The measurements are made in the $A$ $=1$ cell at $\mathrm{Ra}=8.6 \times 10^{9}$. The solid and dashed lines are the linear fits to the measured $\bar{u}$ (open circles) and $\bar{v}$ (open triangles), respectively.

\section{B. Rayleigh number dependence}

In the above, we discuss the velocity measurements at a fixed value of Ra. We now show how the velocity profile changes with the Rayleigh number. We focus our attention on the velocity measurements in the $A=1$ cell with Ra varied in the range $10^{9} \leqslant \mathrm{Ra} \leqslant 10^{10}$. Figure 9 shows the timeaveraged velocity components $\bar{u}, \bar{v}$ (open symbols), and their standard deviations, $\sigma_{u}$ and $\sigma_{v}$ (closed symbols), as a function of $\rho_{z}=z / L$. The measured velocity profiles at $\mathrm{Ra}$ $=8.6 \times 10^{9}$ show a structure similar to those measured at $\mathrm{Ra}=3.7 \times 10^{9}$ (see Fig. 2). The transversal velocity component $\bar{w}$ is found to be zero and the flow field in the rotation plane has the same quasi-2D single-roll structure. These measurements reveal that the LSCR shown in Figs. 2 and 3 is invariant with Ra.

Figure 10 shows the time-averaged velocity profile $\bar{u}\left(\rho_{z}\right)$ and the rms velocity profile $\sigma_{u}\left(\rho_{z}\right)$ in the vertical scan at three different values of $\mathrm{Ra}$. It is seen that the measured $\bar{u}\left(\rho_{z}\right)$ for different values of Ra can all be brought into coincidence, once $\bar{u}\left(\rho_{z}\right)$ is scaled by its maximum value $u_{\max }$. Plots of $\bar{u}\left(\rho_{z}\right) / u_{\max }$ vs $\rho_{z} / L$ remain invariant and only $u_{\max }$ changes with Ra. Similar scaling behavior is also observed for the rms velocity profile $\sigma_{u}\left(\rho_{z}\right)$. Figure 10 thus suggests that the flow structure in a given cell is invariant with $\mathrm{Ra}$. While the measured velocity profiles over varying $\mathrm{Ra}$ in the same cell scale with each other, the velocity profiles obtained from different cells with varying aspect ratios do not scale. It is shown in Sec. III A that the shape of the LSCR and its tilt angle $\alpha$ vary with the aspect ratio of the cell (see Fig. 15). Therefore, we conclude that the spatial structure of the LSCR scales with Ra but its exact form depends on the shape of the convection cell.

Because of its scaling behavior, the LSCR can be characterized by a single parameter. It is either the maximum rota-
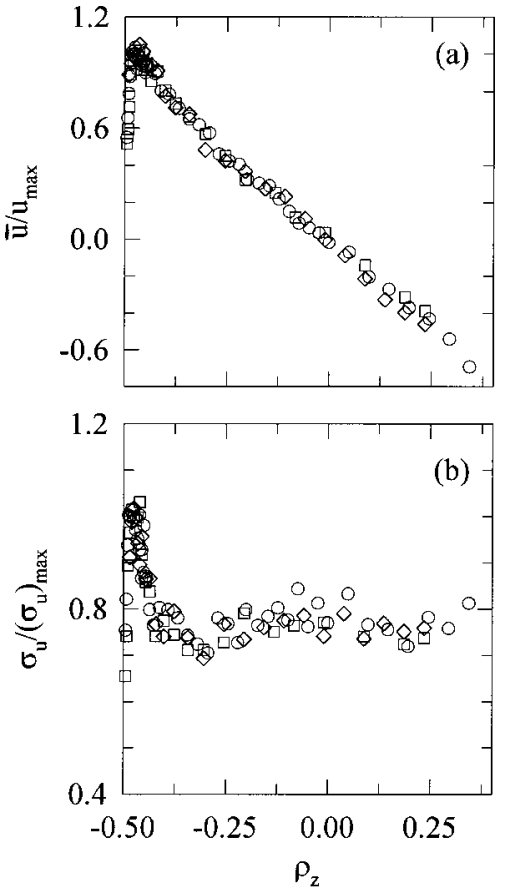

FIG. 10. (a) Normalized velocity component $\bar{u} / u_{\max }$ and (b) its standard deviation $\sigma_{u} /\left(\sigma_{u}\right)_{\max }$ as a function of the normalized vertical position $\rho_{z}=z / L$. The measurements are made in the $A=1$ cell at $\mathrm{Ra}=1.7 \times 10^{9}$ (squares), $3.7 \times 10^{9}$ (circles), and $8.1 \times 10^{9}$ (diamonds).

tion speed $\bar{u}_{\text {max }}$ or the rotation rate $\gamma_{u}$ obtained from the slope of the linear longitudinal velocity profile $\bar{u}\left(\rho_{z}\right)$ shown in Fig. 2. In the experiment, $\gamma_{u}$ is determined more accurately because it is obtained by a linear fit to many data points. Because the measured $\bar{u}\left(\rho_{z}\right)$ over varying Ra scales with each other, the Ra dependence of $\bar{u}_{\text {max }}$ must be the same as that for $\gamma_{u}$. Figure 11 shows the normalized rotation rate $\gamma_{u} L^{2} / \kappa$ (open circles) and the normalized maximum rotation speed $2 u_{\max } L / \kappa$ (closed circles) as a function of Ra. The two

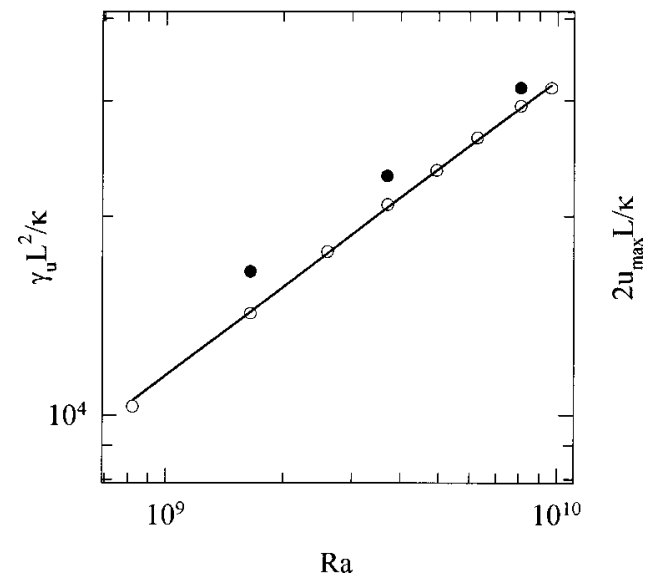

FIG. 11. Normalized rotation rate $\gamma_{u} L^{2} / \kappa$ (open circles) and normalized maximum rotation speed $u_{\max } L / \kappa$ (closed circles) as a function of $\mathrm{Ra}$ in the $A=1$ cell. The solid line shows the power-law fit $\gamma_{u} L^{2} / \kappa=1.1 \mathrm{Ra}^{0.45}$. 


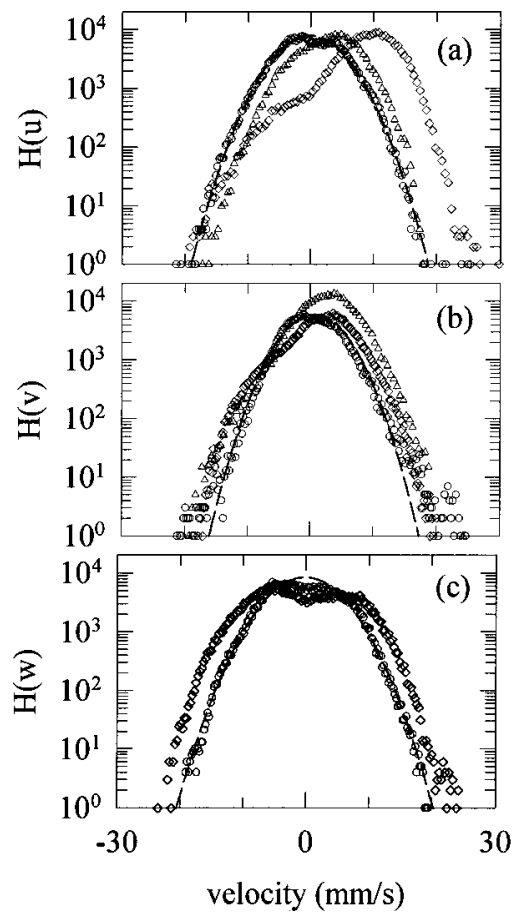

FIG. 12. Measured velocity histograms (a) $H(u)$, (b) $H(v)$, and (c) $H(w)$ at various vertical positions $\rho_{z}=z / L$. The measurements are made in the $A=1$ cell at $\mathrm{Ra}=3.7 \times 10^{9}$. The values of $\rho_{z}$ are: (a) -0.46 (diamonds), -0.15 (triangles), 0 (circles); (b) -0.40 (diamonds), -0.25 (triangles), 0 (circles); and (c) -0.44 (diamonds), and 0 (circles). The dashed curves show the Gaussian fits to the measured $H(u), H(v)$, and $H(w)$ at the cell center.

quantities are normalized in such a way that they become equal when $u_{\max } /(L / 2)=\gamma_{u}$. It is seen that the measured $\gamma_{u} L^{2} / \kappa$ is well described by a power law $\gamma_{h} L^{2} / \kappa=1.1 \mathrm{Ra}^{\epsilon}$ (solid line) with the exponent $\epsilon=0.45 \pm 0.06$. Figure 11 shows that the Ra scaling of the measured $2 u_{\max } L / \kappa$ is indeed the same as that for $\gamma_{u} L^{2} / \kappa$, but its amplitude is $22 \%$ larger than $\gamma_{u} L^{2} / \kappa$.

Using a dual-beam light scattering method, Xin et al. [13] measured the maximum rotation speed and found $2 u_{\text {max }} L / \kappa \simeq 0.8 \mathrm{Ra}^{0.5}$. Because of the limited Ra range, it is difficult to resolve the small difference between the two values of the exponent within the experimental uncertainties. We notice, however, that the value of $\epsilon$ obtained from Fig. 11 is slightly closer to $3 / 7$ or $4 / 9$ than to $1 / 2$ [4,5,32]. We also find that the measured values of $\gamma_{u} L^{2} / \kappa$ and $u_{\max } L / \kappa$ in the other two cells do not scale with the data shown in Fig. 11. This suggests that the absolute value of $\gamma_{u}$ (or $u_{\max }$ ) varies with the aspect ratio of the cell as well.

\section{Velocity statistics}

We now discuss the statistical properties of velocity fluctuations in the $A=1$ cell. Figure 12 shows the measured velocity histograms $H(u), H(v)$, and $H(w)$ at various vertical positions $\rho_{z}=z / L$. It is seen that the measured velocity histograms in the three different directions at the cell center can all be well described by a Gaussian function over an amplitude range of almost 4 decades (dashed curves). The measured $H(u)$ starts to deviate from the Gaussian form and becomes more and more asymmetric when $\rho_{z}$ is moved farther away from the cell center. It is skewed toward the negative side with respect to the mean flow $\bar{u}$, having the largest skewness at the edge of the viscous boundary layer $(|\rho|$ $\simeq 0.46$ ). At this location, $\bar{u}$ reaches maximum. The measured $H(v)$ shows a behavior similar to that of $H(u)$. The negative skewness at large velocities indicates that while the largescale mean flow carries most thermal plumes in one direction, there are still some energetic thermal plumes emitted from the opposite surface moving against the mean flow.

Because there is no mean flow in the direction perpendicular to the rotation plane, the measured $H(w)$ is symmetric and its skewness is approximately zero at all locations. The width of the measured $H(w)$ (i.e., the rms velocity) becomes maximum at the edge of the viscous boundary layer. It is seen from Fig. 12 that both the measured $H(u)$ and $H(w)$ in the central region exhibit two small bumps near the zero mean, indicating that there is a small oscillation in the measured horizontal velocity components. In fact, we have observed this velocity oscillation directly from the time series data [27]. The emergence of the velocity oscillation is an intriguing feature of turbulent convection and we will discuss details of the oscillation in a separate paper [33]. We also measured the velocity histograms in the horizontal scan and find results similar to those shown in Fig. 12. The measured histograms in the other two cells show behavior similar to that in the $A=1$ cell.

\section{Driving force for the large-scale coherent rotation}

The above velocity measurements clearly show that the flow field in all three cells has a quasi-2D single-roll structure. To find the driving force for the LSCR, we conduct additional flow visualization and temperature measurements in the $A=1$ cell. From the flow visualization we observe that the thermal plumes emitted from the upper and lower surfaces are often sheared to the sidewall region by the largescale horizontal flow near the conducting surface. As a result, warm fluids rise along one side of the cell and cold fluids fall on the opposite side of the cell. Regions occupied by the warm and cold plumes coincide with the buffer layers near the sidewall, in which the time-averaged longitudinal velocity $\bar{u}$ reaches maximum. As shown in Fig. 3, the maximum longitudinal velocity $u_{\max }$ near the sidewall is larger than that near the two conducting surfaces.

It should be noted, however, that $u_{\max }$ also depends on the aspect ratio of the convection cell. In the $A=2$ cell (see Fig. 6 ), the measured $u_{\max }$ near the sidewall is smaller than that near the upper and lower conducting surfaces. This is because the vertical flow near the sidewall occupies larger space than the horizontal flow near the conducting surfaces does. The continuity equation requires that the flow in the two different directions must carry the same amount of fluid. The situation in the $A=0.5$ cell is reversed, and indeed the measured $u_{\max }$ near the sidewall becomes dominant over the horizontal $u_{\max }$ (see Fig. 8). 


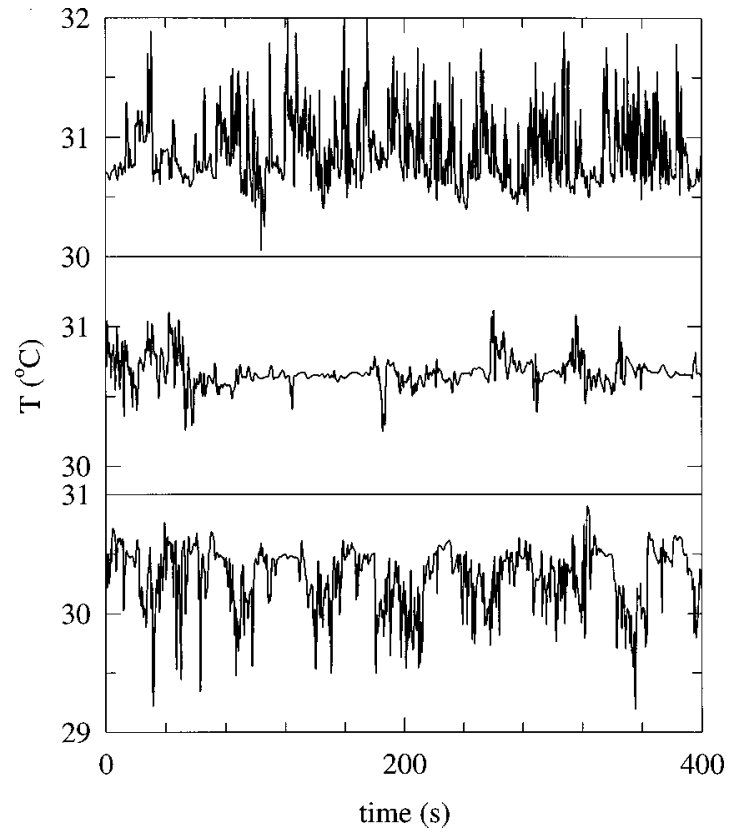

FIG. 13. Time series measurements of the temperature fluctuations at three horizontal positions: $\rho_{x}=+0.42$ (top curve), 0 (middle curve), and -0.42 (bottom curve). These three positions are all located in the rotation plane of the LSCR and at the midheight of the cell. The measurements are made in the $A=1$ cell at $\mathrm{Ra}=3.3 \times 10^{9}$.

From the velocity measurements together with the flow visualization, we arrive at the following driving mechanism for the LSCR. The outer region of the rotation (i.e., the buffer layers around the cell periphery) is the active region that drives the LSCR. Thermal plumes erupt into the region from the upper and lower thermal boundary layers, and many of them are sheared to the sidewall region by the large-scale horizontal flow near the conducting surfaces. The warm and cold plumes, which are separated laterally in the two opposing sidewall regions, exert buoyancy forces to the bulk fluid and drive the vertical flow near the sidewall. This vertical flow in turn enhances the horizontal flow near the upper and lower conducting surfaces. This is a self-feeding process, which connects the thermal plumes with the LSCR. The central core region is "sheared" by the rising and falling plumes near the side wall, resulting in a constant mean velocity gradient in the region. Velocity fluctuations in the central region of the $A=1$ cell are approximately homogeneous and isotropic. They are strong enough to fully mix the thermal plumes that occasionally penetrate into the region.

To further verify this physical picture, we measure the temperature fluctuations at various horizontal positions, $\rho_{x}$ $=x / D$, at the mid-height of the cell. Figure 13 shows the time series measurements at three horizontal positions: $\rho_{x}$ $=+0.42$ (top curve), 0 (middle curve), and -0.42 (bottom curve). These three positions are all located in the rotation plane of the LSCR. Temperature fluctuations at the cell center are characterized by small spikes, which are intermittent in nature and are symmetric about the mean temperature $\bar{T}$. This indicates that the thermal plumes are fully mixed in the

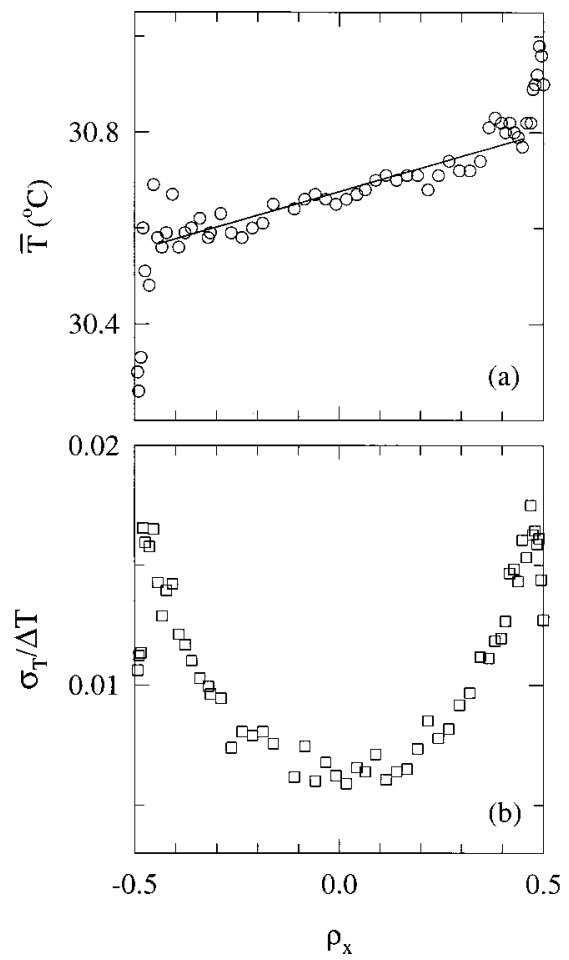

FIG. 14. (a) Time-averaged temperature profile $\bar{T}$ as a function of the normalized horizontal position $\rho_{x}=x / D$. The solid line is a linear fit to the data points in the central region. (b) Normalized rms temperature profile $\sigma_{T} / \Delta T$ as a function of $\rho_{x}$. All the measurements are made in the $A=1$ cell at $\mathrm{Ra}=3.3 \times 10^{9}$.

central region of the cell. Near the sidewall, however, temperature fluctuations are found to be highly skewed toward one direction. Cold fluctuations are superposed on an average base line on one side of the cell and warm fluctuations are found on the other side of the cell. These upward (downward) going spikes are associated with the rising warm (falling cold) plumes in the sidewall region.

The spatial separation of the warm and cold plumes is also reflected in the measured rms temperature profile shown in Fig. 14(b). The measured $\sigma_{T} / \Delta T$ is small and remains constant in the central region of the cell. It becomes larger in the sidewall region and reaches a maximum value at the same horizontal locations as those at which the rising and falling velocities become maximum. The lateral separation of the warm and cold plumes also affects the mean temperature profile in the horizontal scan. As shown in Fig. 14(a), the time-averaged temperature $\bar{T}\left(\rho_{x}\right)$ on the right side of the cell having more warm plumes is higher than that on the left side of cell with more cold plumes. The measured $\bar{T}\left(\rho_{x}\right)$ in the bulk region is well described by a linear function with a slope $d T / d x \simeq 1.3 \mathrm{mK} / \mathrm{mm}$ (solid line).

Because of the rotational symmetry of the LSCR, one would expect that there will be a stabilizing temperature gradient of similar amplitude in the vertical direction. Indeed, Tilgner et al. [11] has observed this stabilizing temperature gradient, $d T / d z \simeq 1.1 \mathrm{mK} / \mathrm{mm}$, at a slightly smaller Rayleigh number, $\mathrm{Ra}=1.1 \times 10^{9}$. Figure 14 thus reveals the cause of 
the small temperature gradient in the turbulent bulk region. It is also seen from Fig. 14(a) that the mean temperature profile changes abruptly near the side wall. This abrupt temperature change of $\sim 0.2^{\circ} \mathrm{C}$ occurs in a thin layer of thickness $\sim 6$ $\mathrm{mm}$, which is comparable to the velocity boundary layer thickness (see Fig. 3). The corresponding temperature gradient is $\sim 33 \mathrm{mK} / \mathrm{mm}$, which is much smaller than the temperature gradient $(\Delta T / 2 \delta) \simeq q \times 10^{3} \mathrm{mk} / \mathrm{mm}$ near the upper and lower conducting surfaces. Clearly, this "thermal boundary layer" is caused by the poor mixing inside the viscous boundary layer near the sidewall.

Recently, Villermaux [34] proposed a model aimed at explaining the emission dynamics of the thermal plumes in turbulent convection. The model assumes that the unstable modes (i.e., the thermal plumes) in the upper and lower thermal boundary layers interact via a delayed nonlinear coupling with a time constant $t_{0} \simeq u / L$, where $u$ is the rising (or falling) speed of the thermal plumes. Because of this delayed coupling, the thermal plumes are excited alternately between the upper and lower boundary layers with a local frequency $f_{0} \simeq\left(1 / 2 t_{0}\right)$. This model explains the experimental finding that the temperature power spectrum has a peak at low frequency, which varies with the rotational speed of the largescale circulation [33].

Villermaux's model focused on the emission dynamics of the thermal plumes and did not discuss the dynamic consequence of the alternating plume emission to the flow field. From the velocity measurements and the visualization discussed above, we now have a complete physical picture, which links the local plume emission to the global flow structure. Because of the buoyancy acceleration, the thermal plumes exert vertical forces on the bulk fluid. An alternating eruption of the thermal plumes, therefore, gives rise to a periodic impulsive force to the fluid. Because of the lateral separation of the warm and cold plumes, the vertical thermal forcing becomes a periodic impulsive torque in the plumedominated sidewall region. It is this periodic torque that drives the LSCR continuously. In a recent experiment [27], Qiu et al. have found a well-defined oscillation in the velocity power spectrum.

\section{E. Thermal plumes in the buffer layer}

From the flow visualization we observe that not all the thermal plumes emitted from the upper and lower surfaces are carried to the sidewall region. Some thermal plumes are mixed in the buffer layer near the conducting surfaces. We therefore refer these two horizontal buffer layers as the mixing layers. In the vertical buffer layers near the sidewall, however, most thermal plumes are carried to the upper (lower) conducting surface by the rising (falling) flow without much turbulent mixing. These two vertical buffer layers are referred to as the advecting layers.

The characteristic length (i.e., the width) of a thermal plume is the thermal boundary layer thickness $\delta$. With this length scale, one finds the thermal diffusion time (i.e., the "life time" of a typical thermal plume) is $\tau_{T} \simeq \delta^{2} / \kappa$ and the viscous diffusion time (i.e., the acceleration time for a typical thermal plume) is $\tau_{v} \simeq \delta^{2} / \nu$. Using the experimental values obtained in the $\mathrm{A}=1$ cell: $\delta \simeq 1 \mathrm{~mm}\left(\right.$ at $\left.\mathrm{Ra}=3.7 \times 10^{9}\right), \kappa$ $\simeq 1.47 \times 10^{-3} \mathrm{~cm}^{2} / \mathrm{s}$, and $\nu \simeq 8.0 \times 10^{-3} \mathrm{~cm}^{2} / \mathrm{s}$ (at $30^{\circ} \mathrm{C}$ ), we have $\tau_{v} \simeq 1.25 \mathrm{~s}$ and $\tau_{T} \simeq 6.8 \mathrm{~s}$. Because of the eruptive nature of the thermal plumes, the plume velocity is comparable to the normal $\mathrm{rms}$ velocity $\sigma_{v}$. If the smallest rms velocity $\sigma_{v} \simeq 2 \mathrm{~mm} / \mathrm{s}$ near the conducting surface [see Fig. 2(b)] is taken as the ascending velocity of a thermal plume during the acceleration, we find the acceleration distance $d_{a} \simeq \sigma_{v} \tau_{v} \simeq 2.5 \mathrm{~mm}$, which is deep inside the viscous boundary layer. Using the mixing length theory [32], we find the maximum rms velocity $\sigma_{v} \simeq \operatorname{Pe}_{c}\left[(L / 2)-\left|z_{1}\right|\right] \kappa / \delta^{2}$ $\simeq 4 \mathrm{~mm} / \mathrm{s}$ at $z_{1} \simeq-92.5 \mathrm{~mm}\left(\rho_{z}=-0.46\right)$. Here we have taken the critical Péclet number $\mathrm{Pe}_{c}=3$ [Ref. [32]]. The calculated $\sigma_{v}$ is very close to the measured value $\sigma_{v} \simeq 4.5$ $\mathrm{mm} / \mathrm{s}$. With the calculated $\sigma_{v}$, we find the corresponding traveling distance $d_{p} \simeq \sigma_{v} \tau_{T} \simeq 30 \mathrm{~mm}$. It is seen from Fig. 2 that the measured vertical velocity $\bar{v}$ reaches maximum at this distance $\left(\rho_{z}=-0.35\right)$.

The above numerical calculation supports the following "life cycle" for an individual thermal plume emitted from the conducting surface. It first accelerates within the viscous boundary layer and then travels through the bulk part of the mixing layer with a terminal velocity comparable to $\sigma_{v}$. Finally, the thermal plume is shattered and mixed with the surrounding fluid in the border region between the mixing layer and the central region, in which the convecting fluid becomes fully mixed. This dynamic process was first discussed in a theoretical model by Castaing et al. [4] It should be pointed out that this mixing dynamics applies only to the individual plumes, whose characteristic size (or width) is comparable to the thermal boundary layer thickness $\delta$. Because their life time $\tau_{T}$ is short, these thermal plumes cannot reach to the opposite conducting surface.

The rising and falling plumes near the sidewall, therefore, must be a new class of plumes, whose life time is longer than $\delta^{2} / \kappa$. From the flow visualization we observe that the thermal plumes in the sidewall region often group together when moving across the cell. Individual plumes are observed rarely near the sidewall. When two thermal plumes combine into one, the characteristic size of the "composite plume" is doubled and thus its life time is quadrupled. In a recent experiment [33], we find that in the $A=1$ cell it takes $t_{0} \simeq 28 \mathrm{~s}$ for a rising plume to reach the upper surface. When two thermal plumes form a "composite plume," its lifetime becomes $\tau_{T} \simeq(2 \delta)^{2} / \kappa \simeq 27.2 \mathrm{~s}$, which is very close to the measured cell crossing time.

From the above observations, we conclude that there are two types of thermal plumes in turbulent convection. Type 1 plumes are individual ones with a short life time, and type 2 plumes are composite ones having a longer life time. For the "composite plumes," the thermal diffusion is not very important and their rising (or falling) velocity (proportional to $\bar{u}$ ) can be estimated by balancing the buoyancy with the viscous drag. At a fixed $\mathrm{Ra}$, we have [4] $u_{\max }$ $\sim \operatorname{Ra} \kappa(n \delta)^{2} / L^{3}$, where $n \delta$ is the typical size of the "composite plume." The exact numerical factor for $u_{\max }$ depends on the shape of the "composite plume." Clearly, the formation of "composite plumes" is a collective behavior of the 


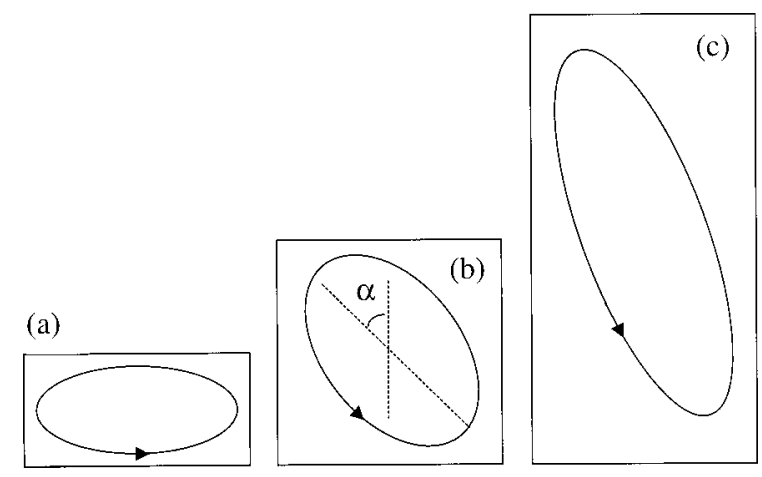

FIG. 15. Sketch of the large-scale quasi-two-dimensional flow structure observed in the (a) $A=2$, (b) $A=1$, and (c) $A=0.5$ cells.

thermal plumes under the influence of the large-scale horizontal flow near the conducting surface. We believe that both types of thermal plumes contribute to the heat transport process in turbulent convection and their relative weight needs to be determined experimentally.

\section{SUMMARY}

We have carried out a systematic study of the large-scale velocity structures in turbulent thermal convection. The experiment is conducted in three different aspect ratio cells filled with water. Laser Doppler velocimetry is used to measure the velocity profiles and statistics over varying Rayleigh numbers and at various spatial positions across the whole convection cell. The results obtained in the aspect-ratio $A$ $=1$ cell are compared with those in the $A=2$ and $A=0.5$ cells.

Large velocity fluctuations are found both in the central region and near the cell boundary. Despite the large velocity fluctuations, the flow field still maintains a large-scale quasi-2D structure, which rotates in a coherent manner. Figure 15 shows the sketch of the coherent single-roll structure observed in the three convection cells. These flow structures scale with $\mathrm{Ra}$ and can be divided into three regions in the rotation plane: a thin viscous boundary layer, a central core region with a coherent rigid-body rotation, and an intermediate buffer region. It is seen from Fig. 15 that the LSCR is not exactly of circular shape. Rather, the rotation is around a tilted ellipse. It is found that the tilt angle $\alpha$ with respect to the vertical direction varies with the aspect ratio of the cell but is not sensitive to Ra. The fact that a quasi-2D single-roll structure is found in all three cells suggests that the LSCR is not the remnant of the convection rolls formed at low Rayleigh numbers, rather, it is a unique new state of turbulent convection.

From the flow visualization and the temperature measurements, we find that the thermal plumes emitted from the upper and lower surfaces are often sheared to the sidewall region by the large-scale horizontal flow near the conducting surface. The warm and cold plumes, which are separated laterally in the two opposing sidewall regions, exert buoyancy forces to the bulk fluid and drive the vertical flow near the sidewall. This vertical flow in turn enhances the horizontal flow near the conducting surfaces, providing a selffeeding mechanism, which drives the LSCR continuously. Under this mechanism, the outer region of the rotation (i.e., the buffer layers around the cell periphery) is the plumedominant active region that drives the LSCR. The central core region is "sheared" by the vertical flow near the sidewall, resulting in a constant mean velocity gradient in the region.

This spatial separation of the active and passive regions has important implications to the physical understanding of turbulent convection. First, it suggests that the boundary condition of the sidewall is important in determining the flow structure in small aspect-ratio cells, such as those used in the present experiment. Second, it affects the scaling of smallscale velocity fluctuations over varying length scales. Recently, several theoretical calculations [35-37] have suggested that the velocity difference $\delta v(\ell)$ in turbulent convection changes its scaling behavior when the separation $\ell$ becomes larger than the Bolgiano scale $\ell_{B}$. At large length scales $\left(\ell>\ell_{B}\right)$, buoyancy is important and one has the Bolgiano-Obukhov scaling: $\delta v(\ell) \sim \ell^{3 / 5}$. At smaller length scales, however, $\delta v(\ell)$ obeys the usual Kolmogorov scaling: $\delta v(\ell) \sim \ell^{1 / 3}$. The experiment indicates that this crossover may vary with the spatial position in the cell.

Finally, the spatial separation of the active and passive regions may also affect the Ra-scaling of the mean and rms velocities. As discussed in Sec. III E, there are two types of thermal plumes in turbulent convection. Individual plumes emitted from the upper and lower surfaces have short life times and some of them are mixed in the horizontal mixing layers. Other thermal plumes, however, group together under the influence of the large-scale horizontal flow near the conducting surface and form "composite plumes." These "composite plumes" have longer life times and thus can travel through the vertical advecting layer and get to the opposite conducting surface. From the flow visualization and the velocity measurements, we find that the large-scale circulation (i.e., the mean flow) is driven mainly by the "composite plumes" near the sidewall, whereas the velocity fluctuations are produced primarily by the individual plumes. Because thermal diffusion is less important to the "composite plumes," their rising (or falling) velocity (proportional to the mean flow) may have a different Ra-scaling from that of the rms velocity. In scaling theories of turbulent convection $[1,2,4]$, the mean velocity of the large-scale flow is often used as the characteristic velocity, whereas the role played by the rms velocity, which is much larger than the mean velocity in the bulk region, has not been studied in great detail.

The present experiment provides an interesting example to demonstrate how otherwise random unstable modes in a closed system are organized in both space and time to generate a large-scale flow structure, which rotates in a coherent manner. The formation of the LSCR makes turbulent convection a unique and ideal system to explore new ways of separating small-scale fluctuations from the large-scale coherent structures in turbulence [38]. 


\section{ACKNOWLEDGMENTS}

We thank Y.-B. Du for providing us the flow visualization data and R. E. Ecke for useful discussions. The assis- tance of M. Lucas and his team in fabricating the convection cells is gratefully acknowledged. This work was supported by the National Science Foundation under Grant No. DMR0071323.
[1] E. Siggia, Annu. Rev. Fluid Mech. 26, 137 (1994).

[2] S. Grossmann and D. Lohse, J. Fluid Mech. 407, 27 (2000).

[3] F. Heslot, B. Castaing, and A. Libchaber, Phys. Rev. A 36, 5870 (1987).

[4] B. Castaing, G. Gunaratne, F. Heslot, L. Kadanoff, A. Libchaber, S. Thomae, X.-Z. Wu, S. Zaleski, and G. Zanetti, J. Fluid Mech. 204, 1 (1998).

[5] B. I. Shraiman and E. D. Siggia, Phys. Rev. A 42, 3650 (1990).

[6] A. M. Garon and R. J. Goldstein, Phys. Fluids 16, 1818 (1973)

[7] J. W. Deardorff and G. E. Willis, J. Fluid Mech. 28, 675 (1967).

[8] D. E. Fitzjarrald, J. Fluid Mech. 73, 693 (1976).

[9] H. Tanaka and H. Miyata, Int. J. Heat Mass Transf. 23, 1273 (1980).

[10] M. Sano, X.-Z. Wu, and A. Libchaber, Phys. Rev. A 40, 6421 (1989).

[11] A. Tilgner, A. Belmonte, and A. Libchaber, Phys. Rev. E 47, R2253 (1993).

[12] Y. Shen, K.-Q. Xia, and P. Tong, Phys. Rev. Lett. 75, 437 (1995).

[13] Y.-B. Xin, K.-Q. Xia, and P. Tong, Phys. Rev. Lett. 77, 1266 (1996).

[14] X.-L. Qiu and K.-Q. Xia, Phys. Rev. E 58, 5816 (1998).

[15] X.-L. Qiu and K.-Q. Xia, Phys. Rev. E 58, 486 (1998).

[16] S. Ashkenazi and V. Steinberg, Phys. Rev. Lett. 83, 3641 (1999); 83, 4760 (1999).

[17] X. Chavanne, F. Chilla, B. Castaing, B. Hebral, B. Chabaud, and J. Chaussy, Phys. Rev. Lett. 79, 3648 (1997).

[18] J. J. Niemela, L. Skrbek, K. R. Sreenivasan, and R. J. Donnelly, Nature (London) 404, 837 (2000).
[19] T. Takeshita, T. Segawa, J. A. Glazier, and M. Sano, Phys. Rev. Lett. 76, 1465 (1996).

[20] K.-Q. Xia, Y.-B. Xin, and P. Tong, J. Opt. Soc. Am. A 12, 1571 (1995).

[21] P. Tong, K.-Q. Xia, and B. J. Ackerson, J. Chem. Phys. 98, 9256 (1993).

[22] A. Belmonte, A. Tilgner, and A. Libchaber, Phys. Rev. Lett. 70, 4067 (1993); Phys. Rev. E 50, 269 (1994).

[23] S. Cioni, S. Ciliberto, and J. Sommeria, J. Fluid Mech. 335, 111 (1997).

[24] Y.-B. Du and P. Tong, J. Fluid Mech. 407, 57 (2000).

[25] L. E. Drain, The Laser Doppler Technique (Wiley, New York, 1980).

[26] S. Ciliberto, S. Cioni, and C. Laroche, Phys. Rev. E 54, R5901 (1996).

[27] X.-L. Qiu, S. H. Yao, and P. Tong, Phys. Rev. E 61, R6075 (2000).

[28] X.-Z. Wu, Ph.D. thesis, University of Chicago, 1991.

[29] X.-Z. Wu and A. Libchaber, Phys. Rev. A 45, 842 (1992).

[30] Y.-B. Du, Ph.D. thesis, Oklahoma State University, 1999.

[31] Y.-B. Du and P. Tong, Phys. Rev. E 63, 046303 (2001).

[32] R. H. Kraichnan, Phys. Fluids 5, 1374 (1962).

[33] X.-L. Qiu and P. Tong, Phys. Rev. Lett. (to be published).

[34] E. Villermaux, Phys. Rev. Lett. 75, 4618 (1995).

[35] V. S. L'vov and G. E. Falkovich, Physica D 57, 85 (1992).

[36] S. Grossmann and V. S. L'vov, Phys. Rev. E 47, 4161 (1993).

[37] E. S. C. Ching, Phys. Rev. E 61, R33 (2000).

[38] P. Holmes, J. L. Lumley, and G. Berkooz, Turbulence, Coherent Structures, Dynamical Systems and Symmetry (Cambridge University Press, Cambridge, UK, 1996). 